\title{
One-Year MR Angiographic and Clinical Follow-Up after Intracranial Mechanical Thrombectomy Using a Stent Retriever Device
}

\author{
F. Eugène, J.-Y. Gauvrit, J.-C. Ferré, J.-C. Gentric, A. Besseghir, T. Ronzière, and H. Raoult
}

\begin{abstract}
BACKGROUND AND PURPOSE: Little is known about the consequences of arterial wall damage that may be due to mechanical endovascular thrombectomy. Our aim was to perform 1-year MR angiographic and clinical follow-up of patients treated with mechanical endovascular thrombectomy using the Solitaire device.

MATERIALS AND METHODS: Patients with stroke treated between August 2010 and July 2012 were prospectively evaluated with a minimum follow-up of 1 year after mechanical endovascular thrombectomy. Angiographic follow-up was performed on a 3T MR imaging scanner and included intracranial artery TOF MRA and supra-aortic artery gadolinium-enhanced MRA. Images were assessed to detect arterial abnormalities (stenosis, occlusion, dilation) and were compared with the final post-mechanical endovascular thrombectomy run to differentiate delayed and pre-existing abnormalities. Clinical evaluation was performed with the mRS and the 36-Item Short-Form Health Survey questionnaire quality-of-life scale.

RESULTS: Thirty-nine patients were angiographically assessed at the mean term of $19 \pm 4$ months. MRA showed intracranial artery abnormalities in 10 patients, including 5 delayed intracranial artery abnormalities in 4 patients (4 stenoses and 1 dilation), 4 cases of pre-existing intracranial artery stenosis, and 2 occlusions. Pre-existing etiologic cervical artery stenosis or occlusion was observed in 2 patients. All these patients remained asymptomatic during the follow-up period. A significant clinical improvement was observed at 1-year follow-up in comparison with 3-month follow-up $(P<.0001)$, with a good outcome achieved in $62.5 \%$ of patients and an acceptable quality of life restored.
\end{abstract}

CONCLUSIONS: One-year follow-up identifies delayed asymptomatic arterial abnormalities in patients treated with the Solitaire device.

ABBREVIATIONS: $\mathrm{FU}=$ follow-up; $\mathrm{MET}=$ mechanical endovascular thrombectomy; SF-36 = 36-Item Short-Form Health Survey Questionnaire; TOAST = Trial of Org 10172 in Acute Stroke Treatment

S

tent-based devices have proved particularly efficient for achieving fast, safe, and successful recanalization in proximal artery occlusion, ${ }^{1}$ with recanalization rates of approximately $80 \%-90 \%$ by using the Solitaire device (Covidien, Irvine, California). ${ }^{1-5}$ Until now, evaluation of mechanical endovascular thrombectomy (MET) safety and efficacy has mainly relied on shortterm 3-month clinical follow-up, but no data have been available about angiographic arterial changes related to MET procedures or

Received February 8, 2014; accepted after revision April 21.

From the Departments of Neuroradiology (F.E., J.-Y.G., J.-C.F., H.R.), Clinical Pharmacology (A.B.), and Neurology (T.R.), Centre Hospitalier Universitaire, Rennes, France; and Department of Neuroradiology (J.-C.G.), Centre Hospitalier Universitaire, Brest, France.

Please address correspondence to Hélène Raoult, MD, 2 Rue Henri Le Guilloux, 35000 Rennes, France; e-mail: helene.raoult@chu-rennes.fr

三 Indicates article with supplemental on-line table.

http://dx.doi.org/10.3174/ajnr.A4071 stroke etiology and their possible correlation with clinical changes on long-term follow-up (FU).

In animal models, MET was shown to be responsible for vascular damage, ${ }^{6,7}$ depending on the device type, proving less frequent with wall-contact devices such as stent retrievers than with aspiration ones. ${ }^{6}$ The only published short-term angiographic follow-up study after MET by using various devices reported $0.9 \%$ arterial occlusion and $3.4 \%$ de novo stenosis. All cases were asymptomatic and identified as a consequence of arterial wall damage likely due to $\mathrm{MET}^{8}{ }^{8}$ Yet arterial stenosis may evolve with time and lead to clinical decline. It, therefore, seems relevant to assess angiographic vascular status and changes and to correlate any changes with clinical outcomes in patients treated with stent retrievers.

The purpose of this study was to perform 1-year 3T MR angiographic FU of patients treated with a Solitaire device to identify potential wall damage. Angiographic data were correlated with 
clinical FU data on the basis of both functional and quality-of-life evaluation criteria.

\section{MATERIALS AND METHODS Patient Identification}

Ethics approval for this study was granted by the institutional review board after review of the stroke protocol. Informed consent was obtained from all patients before the FU MR imaging. Consecutive patients treated with MET by using a Solitaire stent between August 2010 and July 2012 were included. Angiographic and clinical FU was systematically performed with a minimum follow-up of 1 year after MET.

Patients were eligible for an endovascular procedure according to the institutional stroke protocol if they met the following inclusion criteria: feasibility of thrombectomy within 6 hours of symptom onset for anterior circulation occlusion or within 24 hours for posterior circulation occlusion, proximal thrombus on MR imaging or angio-CT, with no limit in terms of age, initial NIHSS score, or the size of the infarct on imaging.

The exclusion criteria were as follows: comorbidity with poor prognosis (neoplasia, cardiac or respiratory failure) or hemorrhagic event as shown on initial imaging.

Within 4.5 hours of symptom onset, all eligible patients received an intravenous thrombolysis bolus according to the generally accepted inclusion and exclusion criteria. MET was performed either immediately after intravenous thrombolysis ("combined therapy"), 1 hour after initiation of intravenous thrombolysis if no clinical improvement was observed ("rescue therapy"), or otherwise as "stand-alone" therapy when the patient was not eligible for intravenous thrombolysis. ${ }^{3}$ Combined or rescue therapy was performed according to the time from symptom onset and the location of the occlusion, as in the REscue, COmbined and Stand-alone Thrombectomy (RECOST) study, ${ }^{3}$ or the terms of the protocol if the patient was enrolled in a randomized trial.

\section{Treatment Protocol}

All procedures were performed by a neurointerventionalist on a biplanar system (Allura; Philips Healthcare, Best, Netherlands) with the patient under general anesthesia, with a target systolic blood pressure of above $140 \mathrm{~mm} \mathrm{Hg},{ }^{9}$ and by using a femoral artery approach. For anterior circulation occlusions, a $6 \mathrm{~F}$ or $8 \mathrm{~F}$ guide catheter (Envoy; Codman \& Shurtleff, Raynham, Massachusetts) was inserted into the target carotid artery. We preferred $8 \mathrm{~F}$ guide catheters to achieve fast and effective recanalization when the procedure was performed by a lone practitioner. For posterior circulation occlusions, a $6 \mathrm{~F}$ guide catheter was inserted into the dominant, or navigable, vertebral artery. The guide catheter was injected with nimodipine (15-mL nimodipine [Nimotop]/L of sodium chloride). A 0.021-inch inner lumen Rebar-18 microcatheter (Covidien) was carefully navigated through the thromboembolic occlusion over a 0.014-inch steerable Transend microwire (Stryker, Kalamazoo, Michigan) under fluoroscopic control. A microcatheter angiographic run was subsequently performed to confirm the correct position of the microcatheter distal to the thrombus. The microwire was replaced by the Solitaire FR (Covidien) mechanical thrombectomy retrievable stent $(4 \times 15$ $\mathrm{mm}$ or $4 \times 20 \mathrm{~mm}$ ). The Solitaire FR was fully deployed, and angiographic runs were performed to check for correct placement and expansion of the device and flow restoration. The fully deployed Solitaire FR was maintained in place for 5-7 minutes, and the stent and microcatheter were then removed. During retrieval, manual aspiration was performed with a $60-\mathrm{mL}$ syringe to reverse the flow and remove any clot debris in the guide catheter lumen. If necessary, up to 5 Solitaire passes were performed.

Neither intra-arterial rtPA nor any device other than Solitaire was used.

In the case of underlying arterial stenosis at the cerebral occlusion site or in the involved cervical artery, additional angioplasty by using a balloon and possibly permanent stent placement was performed at the discretion of the operator.

All patients were admitted to the intensive stroke care unit for at least 24 hours, where neurologic deficits were monitored and care followed the European Guidelines (http://www.eso-stroke. org) on acute stroke care. Secondary preventive treatments were administered according to the applicable European Guidelines.

\section{Baseline Neuroradiologic Evaluation and Follow-Up}

The baseline neuroradiologic data retrieved included the following: infarct extension on pretreatment imaging (lesions were considered severe if the DWI-ASPECTS was $<5$ for anterior circulation occlusion ${ }^{3}$ or $>3$ according to the scale of Renard et $\mathrm{al}^{10}$ for posterior circulation occlusion), details of the recanalization procedure (number of passes; occurrence of dissection, vasospasm, or extravasation; and procedural time defined as the interval between femoral puncture and the last angiographic run), and time to recanalization (between symptom onset and the first Solitaire deployment ${ }^{4}$ ). Good recanalization after MET was defined with a Thrombolysis in Cerebral Infarction score of $2 \mathrm{~b}$ or $3 .^{11}$

A CT scan was obtained immediately after the procedure in the angiography suite to screen for immediate hemorrhagic transformation or any unforeseen procedural complications and after 24 hours to detect intracranial hemorrhage. ${ }^{12}$

One-year FU MR imaging was prospectively performed from July 2012 to January 2013 on a 3T system (Magnetom Verio; Siemens, Erlangen, Germany) including DWI, axial FLAIR, highresolution arterial wall axial TSE T2WI $($ TE/TR $=80 / 3000 \mathrm{~ms}$, $\mathrm{FOV}=180$, voxel $=0.4 \times 0.5 \times 1.7$, time of acquisition $=4$ minutes 48 seconds), axial gradient-recalled echo T2 sequences, $3 \mathrm{D}$ $\mathrm{TOF}(\mathrm{TE} / \mathrm{TR}=3.5 / 25 \mathrm{~ms}, \mathrm{FOV}=200$, voxel $=0.4 \times 0.6 \times 0.5$, time of acquisition $=3$ minutes 46 seconds), and 3D BolusTrak gadolinium-enhanced supra-aortic artery MR angiography (FOV = 320 , voxel $=0.7 \times 0.7 \times 0.7$, time of acquisition $=0$ minutes 44 seconds). ${ }^{13,14}$

Indeed, MRA is a noninvasive imaging tool and, at 3T, has proved reliable for detecting intracranial stenosis in comparison with DSA. ${ }^{15}$ MR images were analyzed in consensus by 2 experienced neuroradiologists (J.-Y.G., H.R.) to detect stroke recurrence or hemorrhagic lesions in comparison with pretreatment MR imaging. MRA images (gadolinium-enhanced supra-aortic artery and $3 \mathrm{D}$ TOF) were analyzed together to 


\begin{tabular}{|c|c|c|c|}
\hline & Population $(n=40)$ & $\operatorname{ACO}(n=30)$ & $\operatorname{PCO}(n=10)$ \\
\hline \multicolumn{4}{|l|}{ Basic data } \\
\hline Age (yr), mean (SD; range) & $57.4(15.2 ; 27-79)$ & $60(14.9 ; 35-79)$ & $49.6(14.1 ; 27-71)$ \\
\hline Female sex (\%) & $12(30 \%)$ & 10 & 2 \\
\hline Pretreatment NIHSS score, mean (SD) & $15.9(5.5)$ & $16.1(4.9)$ & $15.5(7.3)$ \\
\hline \multicolumn{4}{|l|}{ Premorbid status } \\
\hline Charlson Index $\geq 3$ (\%) & $4(10)$ & 3 & 1 \\
\hline \multicolumn{4}{|l|}{ Etiology } \\
\hline TOAST I-large-artery atherosclerosis & 10 & 5 & 5 \\
\hline TOAST II—cardiac embolism & 16 & 14 & 2 \\
\hline TOAST IV_-other determined etiology & 4 & 3 & 1 \\
\hline TOAST V—undetermined etiology & 9 & 7 & 2 \\
\hline \multicolumn{4}{|l|}{ Initial imaging } \\
\hline CT & 8 & 8 & 3 \\
\hline MRI & 32 & 25 & 7 \\
\hline DWI-ASPECTS $<5$ & 5 & 5 & \\
\hline \multicolumn{4}{|l|}{ Target-vessel territories } \\
\hline Ml segment of the MCA & 29 & 29 & \\
\hline M2 segment of the $M C A$ & 1 & 1 & \\
\hline Basilar artery & 10 & & 10 \\
\hline \multicolumn{4}{|l|}{ Recanalization strategy } \\
\hline Stand-alone & 21 & 14 & 7 \\
\hline Bridging & 10 & 8 & 2 \\
\hline Rescue & 9 & 8 & 1 \\
\hline \multicolumn{4}{|l|}{ Endovascular therapy } \\
\hline Solitaire passes, mean (range) & $2.0(1-7)$ & $1.9(1-4)$ & $2.4(1-7)$ \\
\hline Procedural time (min), mean (SD, range) & $75(40,30-189)$ & $69(30,30-150)$ & $96(54,30-189)$ \\
\hline Time to recanalization (min), mean (SD, range) & $322(127,127-793)$ & $288(90,67-430)$ & $421(170,150-793)$ \\
\hline
\end{tabular}

Note:-ACO indicates anterior circulation occlusion; PCO, posterior circulation occlusion.

detect arterial abnormalities, ${ }^{8}$ including stenosis, occlusions, and dilation at the original site of cerebral occlusion and in the involved cervical artery. Images were compared with the final post-MET run to differentiate delayed and pre-existing abnormalities.

\section{Baseline Clinical Evaluation and Follow-Up}

The following data were retrieved from the medical files: age, sex, pre-existing comorbidity (considered high if the Charlson Comorbidity Index was $\geq 3$ ), vascular risk factors, clinical presentation, stroke etiology according to Trial of Org 10172 in Acute Stroke Treatment (TOAST) criteria, and recanalization strategy. One-year clinical FU was performed by a vascular neurologist (T.R.) on the same day as MR imaging, by using the mRS score, and it was compared with the outcome of the 3-month clinical FU previously performed by the same vascular neurologist. Good outcome was defined as an $\mathrm{mRS}$ score of $\leq 2$, and improvement or decline of at least 1 point on the mRS score defined a change in clinical outcome. ${ }^{16}$ A patient was considered symptomatic during $\mathrm{FU}$ if he or she presented with clinical TIA or stroke recurrence with a permanent deficit.

The previously completed mailed 36-Item Short Form Health Survey Questionnaire (SF-36) was retrieved during the visit. This questionnaire assesses 8 domains on physical, emotional, and mental functioning; is widely used to measure health-related quality of life; and has been validated for patients following a stroke. ${ }^{17}$

\section{Statistical Analysis}

Statistical analysis was performed by using MedCalc for Windows (MedCalc Software, Mariakerke, Belgium). The change in the
mRS score between 3-month and 1-year FU was compared by a $\chi^{2}$ test, ${ }^{16}$ according to 4 categories (mRS $0-1 ;$ mRS 2, 3, and 4-6). ${ }^{16}$ To identify independent predictors of angiographic and clinical outcome at 1-year FU, we performed a backward stepwise logistic regression analysis, including all variables, with $P<.2$ (Fisher exact test) in univariate analysis (age, sex, initial NIHSS score, Charlson Index, TOAST criteria, diabetes, hypertension, smoking, use of intravenous thrombolysis, DWI lesion severity, time to recanalization, procedure time, number of stent passes).

\section{RESULTS}

\section{Baseline Characteristics of Patients and Recanalization Therapy}

Of the 52 patients treated with endovascular therapy by using a Solitaire device between August 2010 and July 2012, 8 died, 4 were lost to follow-up, and 40 were finally available for angiographic assessment at 1-year FU (Table 1). Good recanalization (TICI $2 \mathrm{~b}-3$ ) was achieved in 39/40 patients. Three symptomatic hemorrhages $^{12}$ occurred $(5.8 \%)$ as shown on the 24 -hour CT. These were not visible on the CT scan obtained in the angiography suite and therefore were presumed not to be directly related to the procedure itself.

\section{MR Angiographic Follow-Up}

One-year angiographic FU was available for 39 of the 40 survivors because 1 patient refused the MR imaging. The mean time from stroke to angiographic FU was $19 \pm 4$ months. The 1 -year FU was delayed for a few months for some patients due to their unavailability. On morphologic sequences, in 32 patients investigated with MR imaging before MET, the comparison between pretreat- 

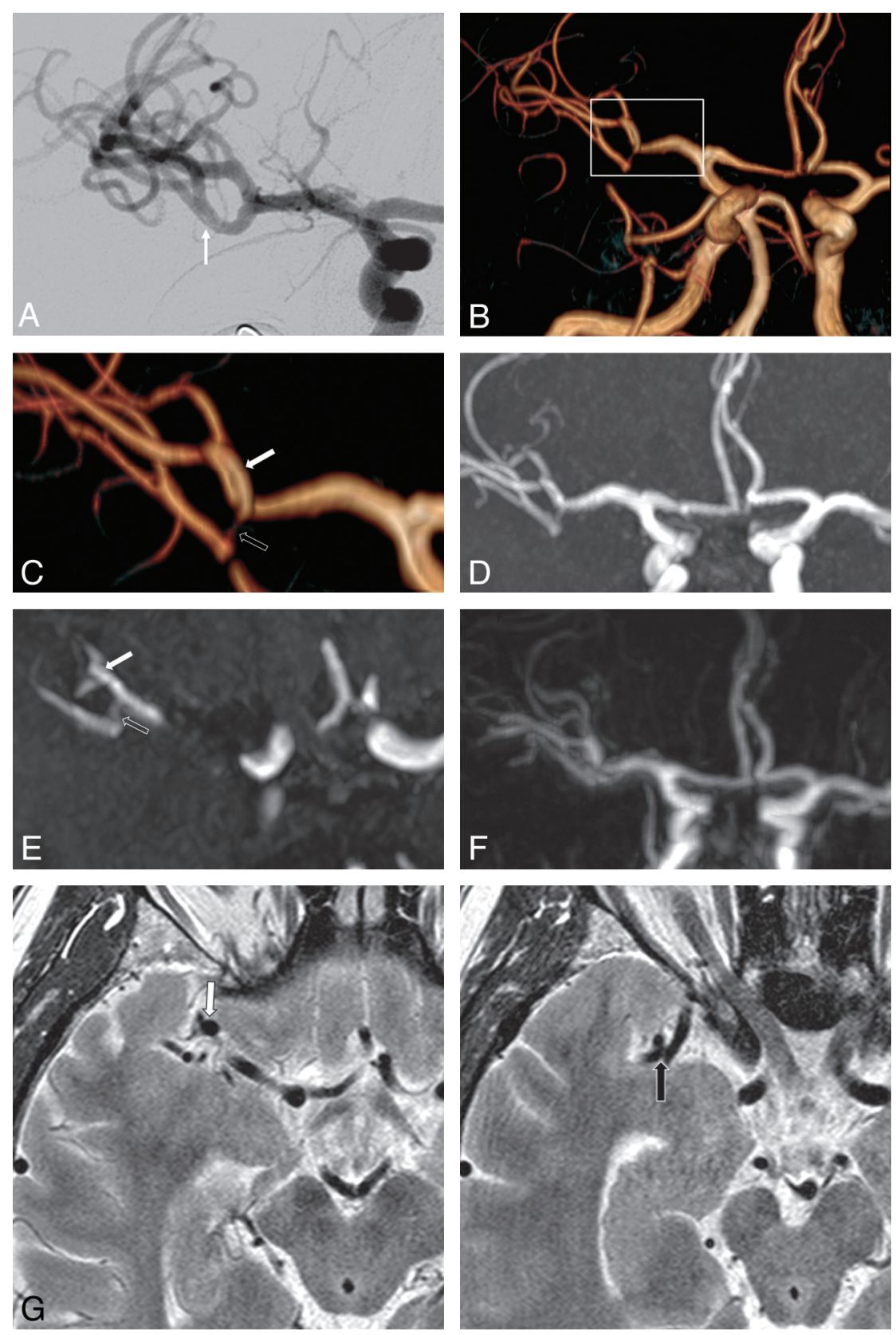

FIG 1. Eighteen-month MR imaging follow-up of de novo arterial stenosis and dilation (patient 2). Final post-MET angiographic run $(A)$ of a 53 -year-old patient treated for a right $\mathrm{M} 1$ middle cerebral artery occlusion, with 5 Solitaire passes in the superior and inferior M2 MCA branches, shows a flap in the inferior stenosis suggestive of dissection (arrow). TOF volume-rendering ( $B$ and $C)$, MIP $(D)$, native image $(E)$, gadolinium-enhanced MRA $(F)$, and high-resolution $T 2(G)$ reveal an asymptomatic dilation (white arrow) of the superior M2 MCA branch and $>50 \%$ stenosis (black arrow) of the inferior M2 MCA branch of the target vessel. Wall analysis at the stenosis is difficult on high-resolution $\mathrm{T} 2$ due to the vertical orientation and the size of the stenosed arterial segment.

ment DWI and long-term FLAIR showed lesion extension for 7 patients $(21.9 \%)$ without any stigmata of a new infarct in other territories, lesion reduction for 4 patients $(12.5 \%)$, and lesion stability for 21 patients $(65.6 \%)$.

MRA showed $10 / 39$ patients (25.6\%) presenting with intracranial artery lumen abnormalities, which are summarized in the On-line Table. Four patients (10.3\%) (patients 1-4) had delayed de novo stenosis of a target vessel, which appeared normal on the final post-MET run. The number of stent passes was 1 for 2 pa- tients and 4 for 2 patients. For 1 of these patients, de novo $>50 \%$ stenosis of the inferior M2 branch coexisted with dilation of the superior M2 branch, compared with the final post-MET run, which showed no stenosis but a suggestive dissection flap on the inferior M2 branch (Fig 1). Four patients (10.3\%) had stenosis of a target vessel, which pre-existed the final post-MET run (patients 5-8). For 3 of these patients, intracranial stenosis was considered as the stroke etiology and stenosis was stable. For 1 of these patients (patient 5), the final post-MET run and long-term FU showed stable $>50 \%$ stenosis, whereas atrial fibrillation was considered the stroke etiology (Fig 2). Last, 2 patients had occlusions (patients 9 and 10) of a target vessel, which were due to recanalization failure. Cervical artery abnormalities were seen for 1 patient (patient 11) with vertebral stenosis and in another (patient 12) with occlusion of the carotid dissection site, regarded as the etiology of the stroke.

Medical secondary prevention drugs for these 12 patients were the following: 7/12 (58.3\%) on statins, 5/12 (22.7\%) on oral anticoagulation, $9 / 12(75.0 \%)$ on platelet inhibitors, and 4/12 (33.3\%) on antihypertensive medication.

\section{Correlation with Clinical Follow-Up}

One-year clinical FU was obtained for 40 survivors. All angiographic lesions were clinically asymptomatic. One patient experienced a recurrent TIA (2\%) without associated arterial MRA abnormalities. The comparison of 3-month and 1-year outcomes showed significant clinical improvement $(P<.001)$, with good clinical outcomes achieved in $50.9 \%$ of patients at short-term FU, rising to $62.5 \%$ at 1 -year FU.

Detailed mRS scores are presented in Fig 3, and SF-36 scores, obtained for 38 of the 40 one-year survivors, are provided in Table 2.

\section{Predictors of MR Angiographic and Clinical Long-Term Outcomes}

No independent factor was identified as predicting arterial abnormality occurrence or mRS at 1-year FU. The number of stent retriever passes did not emerge as a relevant predictor $(P=$ .22). However, 2 independent predictors were associated with a higher SF-36 score after backward stepwise logistic regression: age younger than 65 years $(P=.005 ; 95 \% \mathrm{CI},-28.634$ to -5.454$)$ and time to recanalization $\leq 4.5$ hours $(P=.006 ; 95 \% \mathrm{CI},-28.509$ to -5.329$)$. 

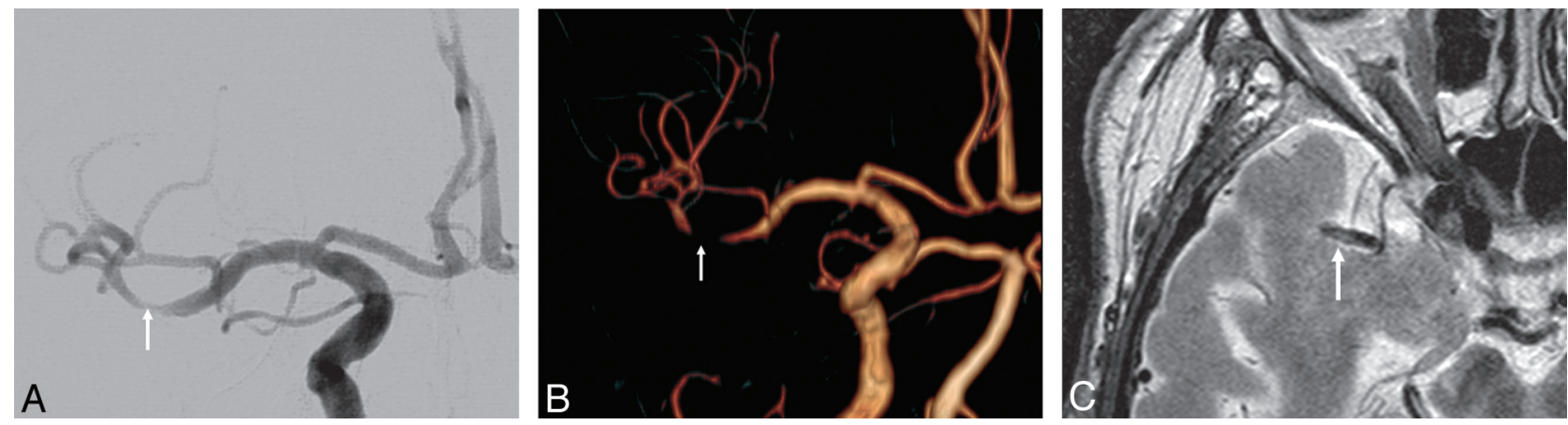

FIG 2. Seventeen-month MR imaging follow-up of pre-existing arterial stenosis (patient 5). Final post-MET angiographic run ( $A$ ) of a 77-year-old patient treated for a right M1 middle cerebral artery occlusion with 2 Solitaire passes, 1 in the superior and 1 in the inferior M2 MCA branch, shows $>50 \%$ stenosis in the inferior M2 MCA division (arrow). TOF volume-rendering (B) shows persistent stenosis (worsening aspect was considered due to TOF stenosis overestimation). High-resolution T2 MRA (C) shows M2 posterior wall thickening. Atrial fibrillation was established as the etiology of the stroke.

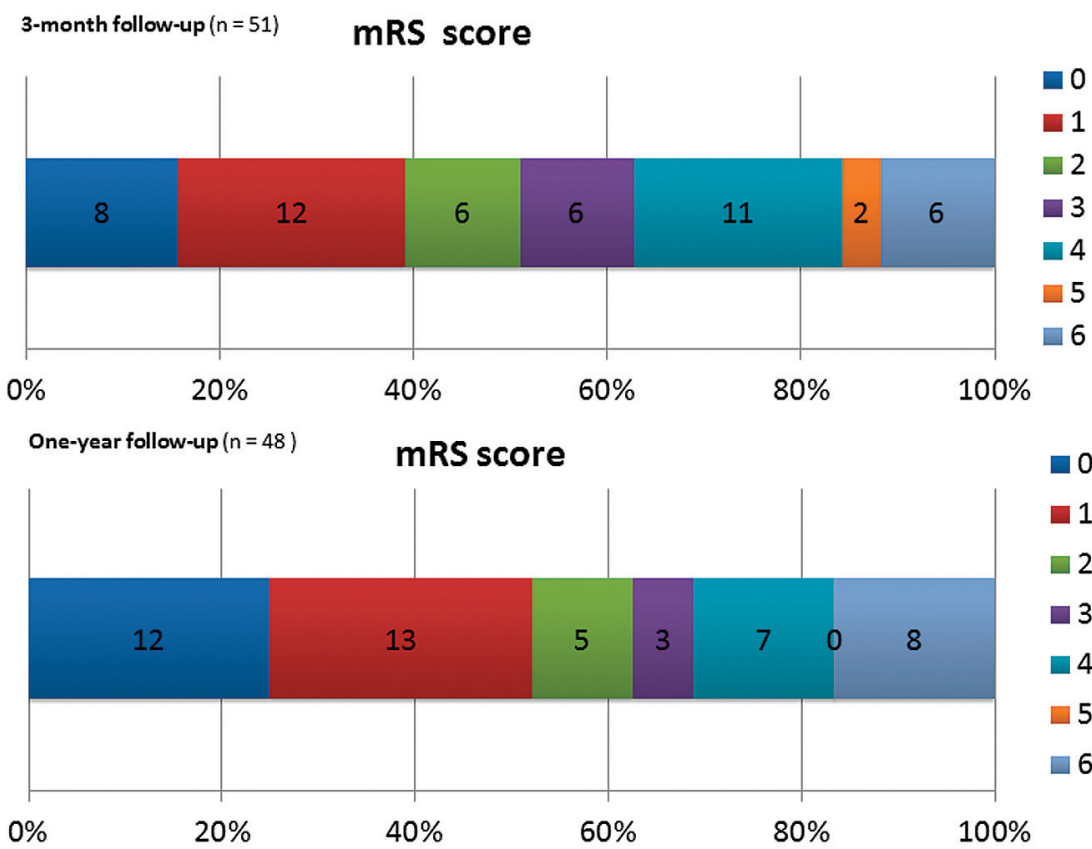

FIG 3. Clinical follow-up. Distribution of 3-month and 1-year mRS (0-6). Values on the bar graphs indicate the number of patients.

Table 2: Clinical outcomes at 1-year follow-up

\begin{tabular}{lccl}
\hline & Population $(\boldsymbol{n}=\mathbf{4 8})$ & \multicolumn{1}{c}{ ACO } & \multicolumn{1}{c}{ PCO } \\
\hline mRS 0-1 (\%) & $25(52.1)$ & $17 / 34(50.0)$ & $8 / 14(57.1)$ \\
mRS 0-2 (\%) & $30(62.5)$ & $22 / 34(64.7)$ & $8 / 14(57.1)$ \\
mRS 3 (\%) & $3(6.2)$ & $2 / 34(5.9)$ & $1 / 14(7.1)$ \\
mRS 4-5 (\%) & $7(14.6)$ & $6 / 34(17.6)$ & $1 / 14(7.1)$ \\
mRS 6 (mortality) (\%) & $8 / 48(16.7)$ & $4 / 34(11.8)$ & $4 / 14(28.6)$ \\
SF-36 PCS (mean) (median, & $51.2(52.8,5.6-100)$ & $49.9(51.9,5.6-100)$ & $56.3(60.6,32.9-71.0)$ \\
$\quad$ range) & & & \\
SF-36 MCS (mean) (median, & $51.4(47.1,4.0-98.8)$ & $50.4(47.1,4.0-98.8)$ & $53.9(48.6,32.3-81.1)$ \\
$\quad$ range) & & & \\
\hline
\end{tabular}

\section{DISCUSSION}

Our study shows that 1-year MR angiographic FU could identify delayed arterial stenosis or dilation in $10.3 \%$ of patients. These delayed arterial abnormalities are of unknown pathophysiologic etiology but may illustrate device-related arterial wall damage, which, up to now, was mainly reported in histopathologic animal studies. ${ }^{18,6}$ Pre-existing stenosis did not appear to have worsened at 1-year FU or to be likely after MET. All arterial abnormalities remained asymptomatic during the FU period, and no independent predictor of arterial abnormality was identified. Moreover, considering the patients in this study as a whole, we observed further significant clinical improvement between 3-month and 1-year FU, whereas natural stroke history without treatment suggests stable clinical outcome over time. ${ }^{19}$ This suggests that clinical evaluation would be more appropriate at 1 or 2 years rather than at 3 months.

MR imaging performed for 1-year FU benefits from the accuracy of TOF-MRA sequences at $3 \mathrm{~T},{ }^{20}$ with high-resolution T2 sequences allowing vessel wall analysis. ${ }^{14}$ One-year MR imaging showed intracranial artery abnormalities, stenosis, or dilation in 8 patients (4 with stenosis pre-existing on the final post-MET run, 3 with isolated de novo stenosis, 1 with delayed de novo stenosis associated with arterial dilation), all asymptomatic, as described below.

Three patients had stenosis that preexisted on the final post-MET run and was established as the stroke etiology (patients 6 to 8 ). This low rate of post-MET vasospasm that occurred in our study would suggest the efficacy of subsequent optimal medical treatment alone, at least at this FU point between 1 and 2 years.

Three patients had delayed isolated stenosis, and 1 patient had delayed stenosis associated with additional delayed arterial dilation (patients 1-4). Three patients had a known stroke etiology other than atherosclerosis suggestive of device-related arterial wall damage or unknown physiologic causes. 
One patient (patient 5) had pre-existing stenosis on the final post-MET run, though the established stroke etiology was cardiac embolism. One theory might be a post-MET vasospasm followed by stenosis due to device-related wall damage, but other physiopathologic causes should not be excluded. This was the only case of vasospasm on the final post-MET run, though some studies have reported immediate post-MET vasospasm ${ }^{8,21,22}$ and even indicated that it may predict subsequent stenosis. ${ }^{8}$ This low rate of post-MET vasospasm in our study may be explained by the continuous infusion of nimodipine through the catheter throughout the procedure. All these patients received appropriate medical secondary prevention.

In summary of our angiographic results, among the 39 patients investigated with MRA 1 year after MET by using Solitaire, for 5 patients $(13 \%)$ arterial abnormalities (intracranial artery occlusive disease, stenosis, or dilation) might be related to the stent retriever device but ischemic injury of the vessel itself or unknown pathophysiologic causes may also be advanced. Several animal studies have already observed intima and medial layer damage after MET. ${ }^{6,18}$ Endothelial damage caused by stent trauma is likely to be one of the underlying mechanisms of delayed stenosis observed in patient studies, though no stenosis was reported for animal models, probably due to the lack of available FU data. Our results corroborate the recent single study on angiographic shortterm follow-up after MET in patients, which showed 3.4\% de novo stenosis on digital subtraction angiography ${ }^{8}$ at a median of 3-month FU after MET by using various stent retrievers. The higher stenosis rate in our study may be explained by a possible stenosis overestimation due to TOF imaging. Thus $<50 \%$ of stenosis cases on MRA may have been insignificant on digital subtraction angiography. Moreover, our FU period was longer than that in the previous study (median of 19 months versus 3 months), yet de novo stenosis may occur late. No predictor for delayed stenosis was identified by the logistic regression. Considering that arterial stenosis may evolve with time and lead to clinical decline, though asymptomatic at 1-year FU, our study suggests that clinical FU after MET should be extended beyond 2 years and associated with noninvasive MR angiographic FU to detect and monitor arterial abnormalities and subsequently gain a greater understanding of their physiopathologic involvement.

Our study has some limitations. First, the small sample size warrants caution, and the stroke protocol applied during the inclusion period did not include certain limitations, such as age or size of the infarct, which are actually considered poor prognostic factors. ${ }^{4,23}$ It is also possible that infarct extension could not be revealed as a predictor of outcome on DWI because pretreatment imaging was a CT scan and not MR imaging for $20 \%$ of patients. Second, TOF MRA may overestimate stenosis. Third, the result of $<50 \%$ stenosis was not shown, and stenosis quantification was not confirmed on digital subtraction angiography (the standard of reference). However being noninvasive, MRA allows repeat scans and, at 3T, proved reliable for detecting stenosis in comparison with DSA, with a sensitivity and specificity of $85 \%$ and $95 \%$, respectively, in patients with stenosis of $>50 \% .^{15}$

Last, high-resolution arterial wall TSE T2 sequences are limited because acquisition is performed in only the axial plane. $3 \mathrm{D}$ high-resolution T1 vessel wall imaging should, therefore, be preferred in future imaging FU. Further long-term FU studies (3-5 years) are required to detect clinical symptoms related to arterial stenosis and, therefore, determine whether MR angiographic FU should be recommended in routine practice. Moreover, further studies including larger cohorts with higher statistical power should help to highlight predictors of arterial abnormality occurrence.

\section{CONCLUSIONS}

One-year FU of patients treated with Solitaire identified delayed asymptomatic arterial abnormalities, some of which may be due to stent retriever-related wall damage. No predicting factor for their occurrence was identified.

Disclosures: François Eugène-UNRELATED: Travel/Accommodations/Meeting Expenses Unrelated to Activities Listed: Covidien, Comments: 2012 Congress of European Federation of Neurological Societies. Thomas Ronzière-UNRELATED: Payment for Lectures (including service on Speakers Bureaus): Boehringer Ingelheim; Travel/Accommodations/Meeting Expenses Unrelated to Activities Listed: Boehringer Ingelheim, Genzyme.

\section{REFERENCES}

1. Dávalos A, Pereira VM, Chapot R, et al. Retrospective multicenter study of Solitaire FR for revascularization in the treatment of acute ischemic stroke. Stroke 2012;43:2699-705

2. Dorn F, Stehle S, Lockau H, et al. Endovascular treatment of acute intracerebral artery occlusions with the Solitaire stent: single-centre experience with 108 recanalization procedures. Cerebrovasc Dis 2012;34:70-77

3. Costalat V, Machi P, Lobotesis K, et al. Rescue, combined, and standalone thrombectomy in the management of large vessel occlusion stroke using the Solitaire device: a prospective 50-patient singlecenter study: timing, safety, and efficacy. Stroke 2011;42:1929-35

4. Raoult H, Eugene F, Ferre JC, et al. Prognostic factors for outcomes after mechanical thrombectomy with Solitaire stent. J Neuroradiol 2013;40:252-59

5. Pereira VM, Gralla J, Davalos A, et al. Prospective, multicenter, single-arm study of mechanical thrombectomy using Solitaire flow restoration in acute ischemic stroke. Stroke 2013;44:2802-07

6. Gory B, Bresson D, Kessler I, et al. Histopathologic evaluation of arterial wall response to 5 neurovascular mechanical thrombectomy devices in a swine model. AJNR Am J Neuroradiol 2013; 34:2192-98

7. Park S, Hwang SM, Song JS, et al. Evaluation of the Solitaire system in a canine arterial thromboembolic occlusion model: is it safe for the endothelium? Interv Neuroradiol 2013;19:417-24

8. Kurre W, Perez MA, Horvath D, et al. Does mechanical thrombectomy in acute embolic stroke have long-term side effects on intracranial vessels? An angiographic follow-up study. Cardiovasc Intervent Radiol 2013;36:629-36

9. Davis MJ, Menon BK, Baghirzada LB, et al. Anesthetic management and outcome in patients during endovascular therapy for acute stroke. Anesthesiology 2012;116:396-405

10. Renard D, Landragin N, Robinson A, et al. MRI-based score for acute basilar artery thrombosis. Cerebrovasc Dis 2008;25:511-16

11. Higashida RT, Furlan AJ, Roberts $\mathrm{H}$, et al. Trial design and reporting standards for intra-arterial cerebral thrombolysis for acute ischemic stroke. Stroke 2003;34:e109-37

12. Hacke W, Kaste M, Fieschi C, et al. Randomised double-blind placebo-controlled trial of thrombolytic therapy with intravenous al- 
teplase in acute ischaemic stroke (ECASS II): Second EuropeanAustralasian Acute Stroke Study Investigators. Lancet 1998;352: 1245-51

13. Mühlenbruch G, Das M, Mommertz G, et al. Comparison of dualsource CT angiography and MR angiography in preoperative evaluation of intra- and extracranial vessels: a pilot study. Eur Radiol 2010;20:469-76

14. Li ML, Xu WH, Song L, et al. Atherosclerosis of middle cerebral artery: evaluation with high-resolution MR imaging at 3T. Atherosclerosis 2009;204:447-52

15. Choi CG, Lee DH, Lee JH, et al. Detection of intracranial atherosclerotic steno-occlusive disease with $3 \mathrm{D}$ time-of-flight magnetic resonance angiography with sensitivity encoding at $3 \mathrm{~T}$. AJNR Am J Neuroradiol 2007;28:439-46

16. Jung S, Mono ML, Fischer U, et al. Three-month and long-term outcomes and their predictors in acute basilar artery occlusion treated with intra-arterial thrombolysis. Stroke 2011;42:1946-51

17. Anderson C, Laubscher S, Burns R. Validation of the Short Form 36 (SF-36) health survey questionnaire among stroke patients. Stroke 1996;27:1812-16

18. Nogueira RG, Levy EI, Gounis M, et al. The Trevo device: preclinical data of a novel stroke thrombectomy device in two different animal models of arterial thrombo-occlusive disease. J Neurointerv Surg 2012;4:295-300

19. Fischer U, Mono ML, Zwahlen M, et al. Impact of thrombolysis on stroke outcome at 12 months in a population: the Bern stroke project. Stroke 2012;43:1039-45

20. Pierot L, Portefaix C, Gauvrit JY, et al. Follow-up of coiled intracranial aneurysms: comparison of 3D time-of-flight MR angiography at $3 \mathrm{~T}$ and $1.5 \mathrm{~T}$ in a large prospective series. AJNR Am J Neuroradiol 2012;33:2162-66

21. Jahan R. Solitaire flow-restoration device for treatment of acute ischemic stroke: safety and recanalization efficacy study in a swine vessel occlusion model. AJNR Am J Neuroradiol 2010;31:1938-43

22. Machi P, Costalat V, Lobotesis K, et al. Solitaire FR thrombectomy system: immediate results in $\mathbf{5 6}$ consecutive acute ischemic stroke patients. J Neurointerv Surg 2012;4:62-66

23. Costalat V, Lobotesis K, Machi P, et al. Prognostic factors related to clinical outcome following thrombectomy in ischemic stroke (RECOST study): 50 patients prospective study. Eur J Radiol 2012; 81:4075-82 